\title{
Impaired Mitochondrial and Metabolic Function of Fibroblasts Derived from Patients with Recessive Dystrophic and Junctional Epidermolysis Bullosa
}

\author{
Authors: \\ Ilona Tietzová, ${ }^{1,2}$ Kirk Twaroski, ${ }^{1}$ Cindy Eide, ${ }^{1}$ Julie H. Ostrander, ${ }^{1}$ Peter \\ A. Crawford, ${ }^{1 *}$ Jakub Tolar \\ 1. University of Minnesota, Minneapolis, Minnesota, USA \\ 2. First Faculty of Medicine, Charles University, Prague, Czech Republic \\ *Correspondence to tolar003@umn.edu
}

Disclosure: The authors have declared no conflicts of interest.

Acknowledgements: The authors would like to thank Gail Celio of the University Imaging Center, University of Minnesota, for providing expertise in electron microscopy for this work. This work was supported by the Richard M. Schulze Family Foundation.

Received:

08.01 .20

Accepted:

10.03 .20

Keywords:

Cellular bioenergetics, epidermolysis bullosa (EB), fibroblasts, fractal dimension, mitochondria.

Citation:

EMJ Dermatol. 2020;8[1]:75-83.

\section{Abstract}

Background: Recessive dystrophic epidermolysis bullosa (RDEB) and junctional EB (JEB) are inherited disorders characterised by fragility and blistering of epithelial tissues leading to pain, pruritus, and adherent scarring. The severity and chronic nature of the resultant skin wounds significantly reduces quality and length of life. Current therapies primarily consist of protective bandaging and nutritional supplementation; there is no cure for these disorders. Although the skin fragility results from a lack of C7 protein (RDEB) and laminin-332 (JEB), other serious aspects of these disorders, such as inflammation that interferes with healing and aggressive squamous cell carcinoma, have not been completely elucidated. Recent research has suggested that mitochondrial function plays a significant role in skin healing.

Objective: To evaluate how mitochondrial function differs in patients with RDEB and JEB.

Method: The energy status of RDEB and JEB patient-derived fibroblasts was determined by Seahorse analysis and metabolite production. The energetics and overall morphology of RDEB and JEB patientderived fibroblasts were assayed as a measure of metabolic stress.

Results: EB patient-derived fibroblasts showed impaired oxidative phosphorylation with concomitant compensation by glycolysis. Morphological parameters were altered in RDEB and JEB fibroblasts compared with controls.

Conclusion: This is the first study to describe changes in mitochondrial energy metabolism, metabolic profile, and mitochondrial morphology of EB patients. 


\section{INTRODUCTION}

Epidermolysis bullosa (EB) is a clinically and genetically heterogeneous group of rare inherited disorders, characterised by fragility of epithelial tissues with blistering following minimal mechanical trauma. ${ }^{1}$ Four major subtypes of EB have been described, each defined by a distinct plane of epidermal-dermal separation: EB simplex, junctional EB (JEB), dystrophic EB, and Kindler syndrome. ${ }^{1}$ The most severe forms of $E B$ are recessive dystrophic EB (RDEB) and JEB, which currently have no curative treatment. Blister cleavage presents immediately below the lamina densa in RDEB and at the lamina lucida in

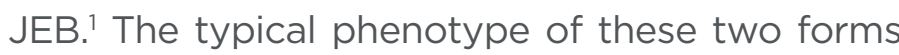
involves mutilating mucocutaneous blistering, chronic cutaneous infection, and aggressive squamous cell carcinoma., ${ }^{1,2}$

The molecular pathology of RDEB results from biallelic loss-of-function mutations in the Type VII collagen gene (COL7A7), resulting in a lack of an extracellular matrix molecule critical for skin integrity. Absent or deficient production of functional Type VII collagen (C7) protein leads to the loss of $\mathrm{C} 7$ homotrimer anchoring fibrils that interact with dermal and epidermal proteins to connect the skin basement membrane to the papillary dermis. One of the most severe forms of JEB is caused by loss-of-function mutations in any of the three genes ( $L A M A 3, \angle A M B 3$, or $\angle A M C 2$ ) composing the heterotrimeric protein laminin-332. This protein interacts with $\mathrm{C} 7$ at the basement membrane to attach the epidermis to the underlying layers through the integrin

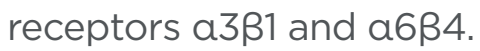

Although genetic mutations have been identified as the origin of these diseases, the complex EB phenotype cannot be completely explained based on the adhesive functions of C7 and laminin-332. For example, the mechanisms responsible for the clinical features of EB, such as photosensitivity and cancer risks, are still poorly understood. ${ }^{3}$

Several pathognomonic features not directly related to skin fragility, such as ageing, inflammation, and squamous cell carcinoma, have been strongly associated with mitochondrial dysfunction and oxidative stress. ${ }^{4-7}$ In addition, there is emerging evidence that mitochondria play an important role in skin physiology and pathophysiology. ${ }^{8}$ Recent research has suggested mitochondrial function promotes keratinocyte and melanocyte differentiation and pigmentation, as well as epidermal progenitor stem cell function. ${ }^{9-11}$

Mitochondria are double-membrane enclosed organelles that generate the majority of cellular energy through the citric acid cycle and oxidative phosphorylation (OXPHOS). ${ }^{12}$ In addition to energy production in the form of ATP, mitochondria are involved in several metabolic pathways including fatty acid $\beta$-oxidation,,$^{13}$ the formation of iron/sulphate clusters, and haem biosynthesis. ${ }^{14}$ They participate in the metabolic processes of lipogenesis, gluconeogenesis, ketogenesis, steroid hormone synthesis, and ammonium detoxification. ${ }^{15-17}$ Mitochondria regulate calcium homeostasis by buffering calcium flux for the plasma membrane, endoplasmic reticulum, and apoptotic pathways. ${ }^{18-20}$

Mitochondria are highly complex and dynamic organelles that can change in number and morphology within a cell during development, cell cycle, or when challenged by various endogenous or exogenous conditions. Defective mitochondrial architecture-function relationships are linked to many human diseases, including metabolic disorders, neurodegenerative disease, ageing, and cancer. ${ }^{21}$

For these reasons, the work presented here focusses on defining the morphologic, bioenergetic, and metabolic characteristics of RDEB and JEB fibroblasts when compared to unaffected fibroblasts. This study showed altered mitochondrial morphology in both forms of EB, and its functional correlation in impaired OXPHOS and subsequent insufficient glycolytic compensation in RDEB and JEB patient-derived fibroblasts.

\section{MATERIALS AND METHODS}

\section{Cell Line Derivation}

Samples were obtained after receiving written informed consent, as approved by the University of Minnesota Institutional Review Board and in adherence with the Declaration of Helsinki. RDEB and JEB patients were screened for diseasecausing mutations as part of the University of Minnesota Epidermolysis Bullosa Center standard 
of care. Primary fibroblasts were obtained from a skin punch biopsy of patients with RDEB (six patients, ranging in age from 1 to 14 years), JEB (five patients, ranging in age from 1 month to 11 years), or unaffected matched donors (two donors aged 9 and 13 years), and derived by mincing the skin tissue that had been immobilised under a sterile coverslip. Biopsies were taken from skin near blistered regions. Cells were grown in Dulbecco's modified Eagle medium (Thermo Fisher Scientific Inc., Waltham, Massachusetts, USA), supplemented with $15 \%$ fetal bovine serum (Thermo Fisher Scientific Inc.), and $0.1 \mu \mathrm{g} / \mathrm{mL}$ each with penicillin and streptomycin (Thermo Fisher Scientific Inc.) at $37^{\circ} \mathrm{C}$ in a humidified $5 \%$ $\mathrm{CO}_{2}$ incubator. The culture medium was changed every third day; all experiments were conducted between passage three and passage 10.

\section{Oxygen Consumption Rate Measurement}

Oxygen consumption rate (OCR) was measured in primary fibroblasts with the XF24e Extracellular Flux Analyzer (Seahorse Bioscience, North Billerica, Massachusetts, USA). Cells were seeded at a density of $35 \times 10^{3}$ cells per well in 24 -well plates and cultured overnight at $37^{\circ} \mathrm{C}$ in a humidified, $5 \% \mathrm{CO}_{2}$ incubator. For the bioenergetics profile, the fibroblasts were consecutively treated with oligomycin A (1.0 $\mu M)$, carbonyl cyanide-4(trifluoromethoxy)phenylhydrazone (FCCP) (0.7 $\mu \mathrm{M})$, and rotenone plus antimycin A (1.5 $\mu M)$. Data were normalised by cell number using the CyQuant ${ }^{\circledast}$ NF Cell Proliferation Assay kit (Thermo Fisher Scientific Inc.). Fluorescence was measured on the SpectraMax ${ }^{\circledast}$ M2 (Molecular Devices, San Jose, California, USA) with an excitation wavelength of $485 \mathrm{~nm}$ and an emission detection wavelength of $530 \mathrm{~nm}$.

\section{ATP/ADP Assay}

Cells were seeded at a density of $1 \times 10^{3}$ cells per well in 96-well plates. Following 24-hour incubation, the EnzyLight ${ }^{\mathrm{TM}}$ ATP/ADP ratio assay was performed (BioAssay Systems, Hayward, California, USA) according to the manufacturer's protocol. The assay was normalised to the total number of cells using the CyQuant NF Cell Proliferation Assay.

\section{Confocal Microscopy}

For all experiments, cells were plated at $2.5 \times 10^{5}$ cells per $6 \mathrm{~cm}$ dish (Thermo Fisher Scientific Inc.) and images were obtained after 48 hours on a Nikon FN1 upright microscope equipped with an A1R scan head and Plan Apo LWD 25x waterimmersion objective lens NA 1.1. Images of different fluorophores were acquired sequentially using the two settings: NADH $405 \mathrm{~nm}$ excitation, 425$475 \mathrm{~nm}$ emission; and flavin adenine dinucleotide (FAD) $488 \mathrm{~nm}$ excitation, 500-550 nm emission. The 12-bit 2048×2048-pixel images were acquired, and final magnification was adjusted by zooming with the laser to attain appropriate pixel size.

\section{Electron Microscopy}

Normal, RDEB, and JEB fibroblasts were collected using Trypsin-EDTA (Thermo Fisher Scientific Inc.) and pelleted. Cell pellets were sent to the University of Minnesota University Imaging Core for processing and imaging. The work was performed using a Philips CM12 Transmission Electron Microscope. Images were taken with the SIA L3C Digital Camera.

\section{Live Cell Imaging}

All images were obtained after 48 hours on a Nikon FN1 upright microscope equipped with an A1R scan head and Plan Apo LWD 25x water-immersion objective lens with NA 1.1 in the chamber at a stable temperature of $37{ }^{\circ} \mathrm{C}$. The cells were stained with MitoTracker ${ }^{\circledR}$ Deep Red FM (Thermo Fisher Scientific Inc.), 640 nm excitation and emission $650-720 \mathrm{~nm}$. Z stacking was performed. 12-bit, $2048 \times 2048$-pixel images were acquired, and the final magnification was adjusted by zooming with the laser to attain appropriate pixel size.

\section{Image Analysis}

For all experiments testing the optical redox ratio, cells were plated at $2 \times 10^{5}$ per $60 \mathrm{~mm}$ glassbottomed dish (MatTek Corporation, Ashland, Massachusetts, USA). Cell images were obtained approximately 48 hours later using a Nikon FN1 upright microscope (UMN Imaging Center). Images of different fluorophores were acquired sequentially using two settings: NADH 405 $\mathrm{nm}$ excitation, 425-475 $\mathrm{nm}$ emission; and FAD $488 \mathrm{~nm}$ excitation, $500-550 \mathrm{~nm}$ emission. The 
pinhole, gain, and offset remained the same for every experiment. For each imaging session, there were two plates of each cell line and two fields of view for both NADH and FAD. Image acquisition took approximately 16 seconds ( $Z$ stack). Following data collection, the NADH/FAD ratio (a measure of the reduction-oxidation ratio) was calculated for every cell in each image. For each acquired NADH and FAD image, ImageJ software (Research Service Brand, National Institute of Mental Health, Bethesda, Maryland, USA) was used to obtain the integrated intensity of NADH and FAD for each cell in the image after the background fluorescence was subtracted. Box fractal dimension was calculated from twodimensional mitochondria images, converted to binary images, then run in built-in box fractal dimension in ImageJ. Box sizes of 2, 3, 4, 6, 8, 12, 16, 32, and 64 pixels were used. For analysis of mitochondrial volume/cell volume ratio, pictures were first deconvoluted using AutoQuant X3 (Media Cybernetics, Rockville, Maryland, USA), then analysed by Imaris 7.7.0 software (Bitplane, Concord, Massachusetts, USA). ${ }^{22}$

\section{L-lactate Assay}

Cells were seeded at a density of $1 \times 10^{3}$ cells per well in 96-well plates. After 48 hours incubation, the L-lactate assay (ScienCell Research Laboratories, Carlsbad, California, USA) was performed according to the manufacturer's protocol. The assay was normalised to cell number using the CyQuant NF Cell Proliferation Assay.

\section{Statistical Analysis}

Results are expressed as mean \pm standard error of the mean (SEM). The significance of the difference between control and experimental conditions was analysed by unpaired Student's t-test; $\mathrm{p}<0.05$ was considered statistically significant.

\section{RESULTS}

\section{Morphologic Changes of Mitochondria in Epidermolysis Bullosa Fibroblasts}

Mitochondrial structure and function are intertwined, and the alteration of one often impacts the other. Transmission electron microscopy was used to image mitochondria in normal, RDEB, and JEB fibroblasts between five and eight matched passages after isolation (Figure 1A-C). Box fractal dimension was used to identify structural changes of the mitochondria in RDEB and JEB fibroblasts compared with control cells. ${ }^{22-24}$ Box fractal dimension is a measure of the texture of an object from twodimensional images derived from a Z stack; it reflects a character of the outer mitochondrial membrane structure whereby a grainier structure correlates to higher fractal dimension. Box fractal dimension increased significantly: $25 \%$ in RDEB and $9 \%$ in JEB fibroblasts compared with nonEB cells (Figure 1D). These observations are consistent with the moderately swollen and more sparsely packed cristae in mitochondria from both EB groups compared with mitochondria of control fibroblasts (mean \pm SEM). Live cell imaging using MitoTracker suggests a more fragmented mitochondrial network with less overall mitochondrial volume in RDEB and JEB fibroblasts compared to controls (Figure 2).

\section{Oxidative Phosphorylation Is Impaired in Epidermolysis Bullosa Fibroblasts}

To examine and quantify different components of mitochondrial function in RDEB and JEB fibroblasts during Seahorse experiments, the cells were analysed in three passages (passages six to nine). After passage 10, an overall decline in respiratory function of all cell types was observed. This may be attributed to telomeredependent senescence. ${ }^{25}$ OCR was measured first in a basal state, then after addition of the ATP synthase (complex V) inhibitor oligomycin, the proton ionophore FCCP, and the respiratory complex I and III inhibitors rotenone and antimycin. Basal respiration was significantly decreased both in RDEB and JEB fibroblasts compared to unaffected fibroblasts derived from healthy individuals (Figure 1E). Respiration measured after treatment with oligomycin reflects decreased OCR to the extent cells are using mitochondria to generate ATP. The remaining $O C R$ is attributed to residual electron transport and oxygen consumption independent of oxidative phosphorylation. Maximal oxygen consumption, measured after addition of FCCP (which collapses the mitochondrial membrane potential), was decreased in JEB fibroblasts (Figure 1F). 

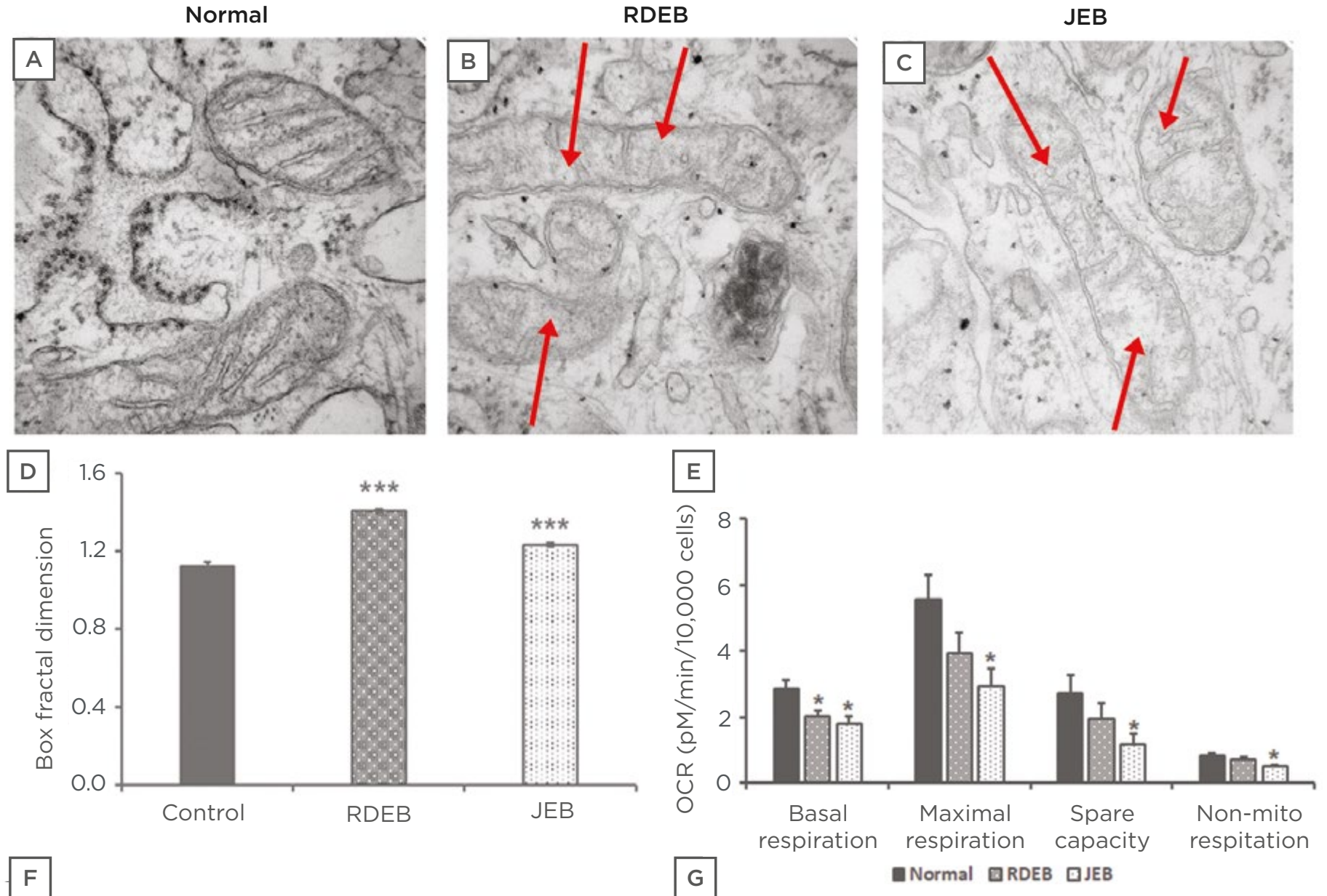

- Normal QRDEB QJEB
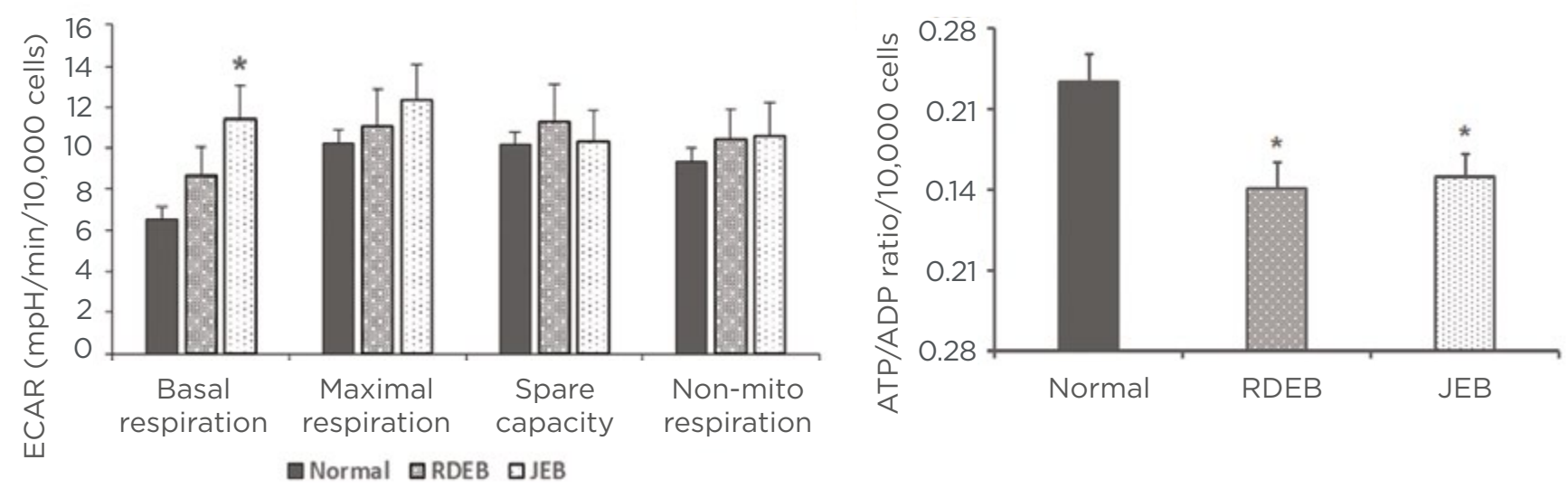

\section{Figure 1: Mitochondria morphology and bioenergetic profile of recessive dystrophic epidermolysis bullosa and junctional epidermolysis bullosa skin fibroblasts.}

TEM of mitochondria in A) normal; B) RDEB; and C) JEB fibroblasts. D) Mitochondrial morphologic changes in RDEB and JEB fibroblasts quantified using box fraction dimension. A total of 30 mitochondria were assayed for each of two normal control, six RDEB, and five JEB patient fibroblast lines (SEM error bars). E) Seahorse X24 measurement of OCR of RDEB and JEB fibroblast lines upon sequential addition of oligomycin (1.O $\mu \mathrm{M})$, FCCP (0.7 $\mu \mathrm{M})$, and rotenone and antimycin (1.0 $\mu$ M each). F) Seahorse X24 measurement of ECAR of normal, RDEB, and JEB fibroblast lines upon sequential addition of oligomycin $(1.0 \mu \mathrm{M})$, FCCP $(0.7 \mu \mathrm{M})$, and rotenone and antimycin (1.0 $\mu \mathrm{M}$ each). G) ADP/ATP ratio measured following 24-hour incubation.

Values represent the means over three passages of fibroblasts from each of two normal control, three RDEB, and three JEB fibroblast lines \pm SEM.

The age ranges for the cell lines: normal (9-13 years), RDEB (1-6 years), and JEB (2-10 months).

Statistical significance was calculated with the Student's t-test: ${ }^{*} \mathrm{p}<0.05 ;{ }^{* * *} \mathrm{p}<0.001$.

ECAR: extracellular acidification rate; FCCP: carbonyl cyanide-4-(trifluoromethoxy) phenylhydrazone; JEB: junctional epidermolysis bullosa; OCR: oxygen consumption rate; RDEB: recessive dystrophic epidermolysis bullosa; TEM:

transmission electron microscopy. 


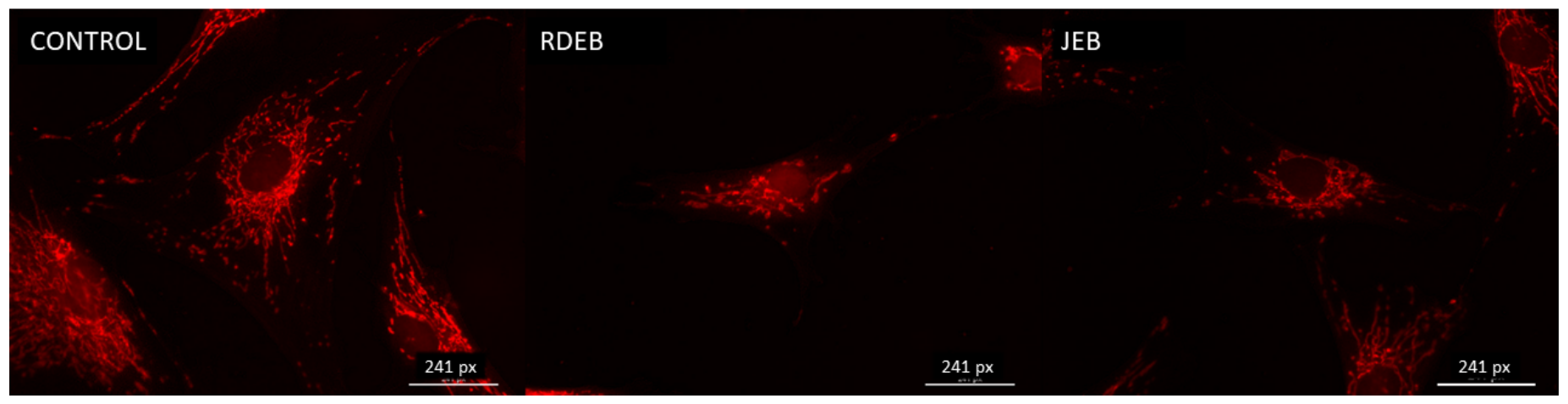

Figure 2: MitoTracker imaging profile of recessive dystrophic epidermolysis bullosa and junctional epidermolysis bullosa skin fibroblasts.

Representative images of control, RDEB, and JEB fibroblasts using the live cell MitoTracker mitochondria stain (bar: 241 pixels).

JEB: junctional epidermolysis bullosa; RDEB: recessive dystrophic epidermolysis bullosa.

Lastly, rotenone and antimycin were injected to inhibit electron flux through complexes I and III of the electron transport chain, causing dramatic suppression of the OCR. The remaining OCR, attributable to $\mathrm{O}_{2}$ consumption in nonmitochondrial organelles, was also significantly decreased in JEB fibroblasts. Moreover, the cellular energy ratio (cytosolic ATP/ADP) of RDEB and JEB fibroblasts was decreased (Figure 1G). These lower cytosolic ATP/ADP ratios determined the cell metabolism to be predominantly glycolytic.

\section{Epidermolysis Bullosa Fibroblasts Compensate Impaired Oxidative Phosphorylation by Glycolysis}

The normalised optical redox ratio was used to further estimate the metabolic profile of EB fibroblasts. ${ }^{26,27} \mathrm{NADH}$ and FAD are the primary electron donor and acceptor of electron transport chain complexes I and II, respectively. ${ }^{28-31}$ Interestingly, mRNA expression profiling of RDEB fibroblasts has found significant differences in the expression of genes involved with nicotinamide metabolism compared to control fibroblasts. ${ }^{32}$ The oxidation-reduction ratio is the most common optical method for measuring cell redox state, as determined by the fluorescence intensity of FAD and NADH (FAD/[FAD+NADH]). 31,33 The redox ratio is strongly associated with $\mathrm{NAD}^{+} / \mathrm{NADH}$ concentration ${ }^{34,35}$ and has been used in vitro and in vivo to track metabolic changes during cell differentiation and malignant transformation. ${ }^{27,36-39}$
In the case of impaired OXPHOS in RDEB and JEB fibroblasts, the authors hypothesised these cells would have a significant difference in redox state compared to unaffected fibroblasts. To test this, confocal microscopy was used to examine a panel of six RDEB, five JEB, and two non-EB fibroblast lines between five and eight matched passages after isolation (Figure 3A). The optical redox ratio of RDEB and JEB fibroblasts was found to be significantly lower than the optical redox ratio of unaffected control cells (Figure 3B), consistent with abnormal respiration in these cells. To determine if glycolytic metabolism is augmented in EB cells with impaired respiration, extracellular acidification rates (ECAR) of RDEB, JEB, and control fibroblasts were measured, and it was observed that ECAR of JEB fibroblasts was significantly higher during basal respiration, but not in RDEB fibroblasts (Figure 1F). ECAR was unchanged in RDEB and JEB fibroblasts relative to normal fibroblasts during maximal and non-mitochondrial respiration. These results could indicate RDEB and JEB fibroblasts are not able to compensate for impaired OXPHOS by increasing glycolysis, although modestly elevated concentrations of L-lactate in the culture media in RDEB and JEB fibroblasts, compared to control fibroblasts, may indicate increased reliance on glycolysis for ATP production under normal oxygen conditions in these cells (Figure 3C). 
A

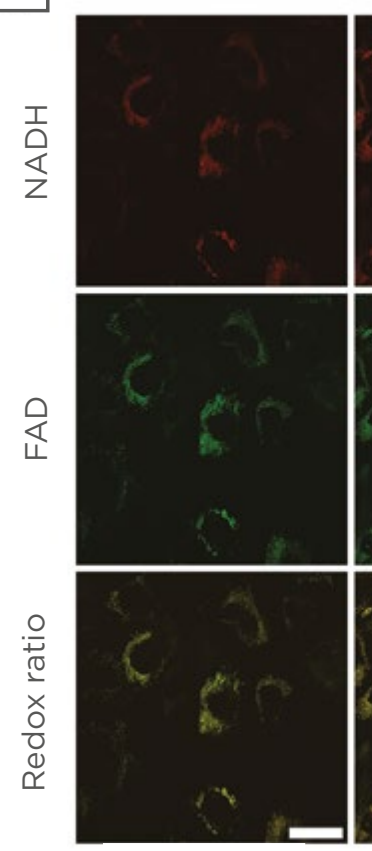

Normal
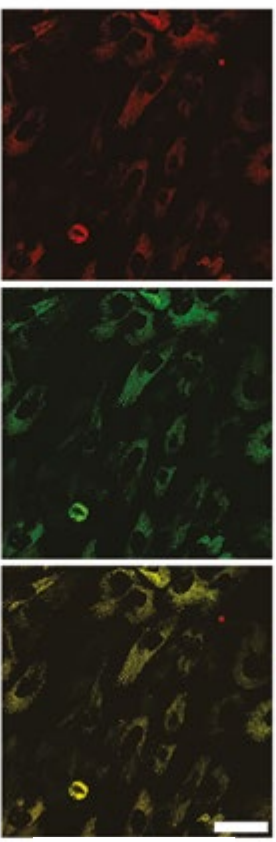

RDEB
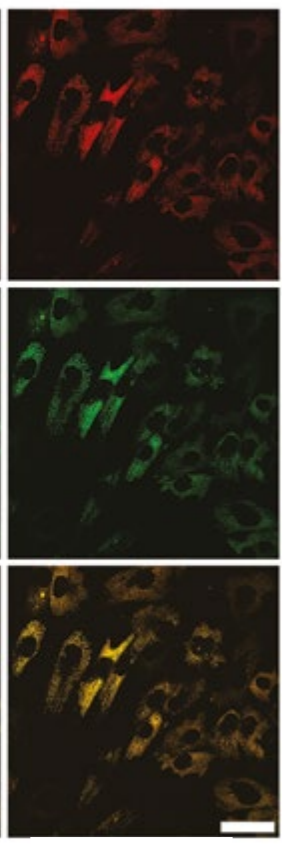

JEB
B

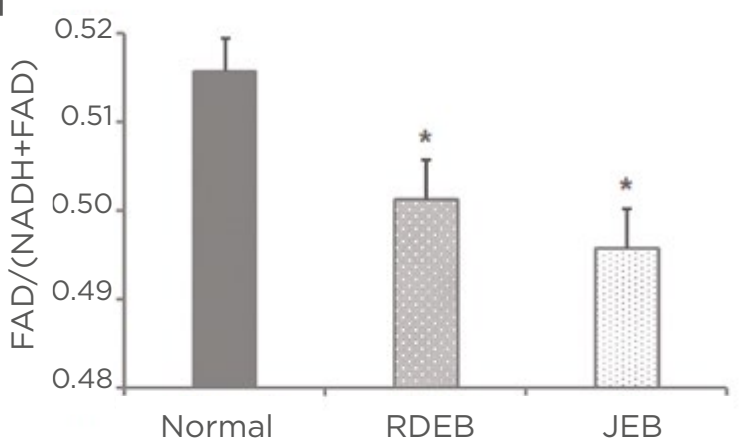

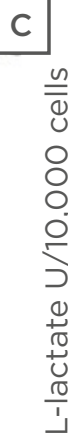

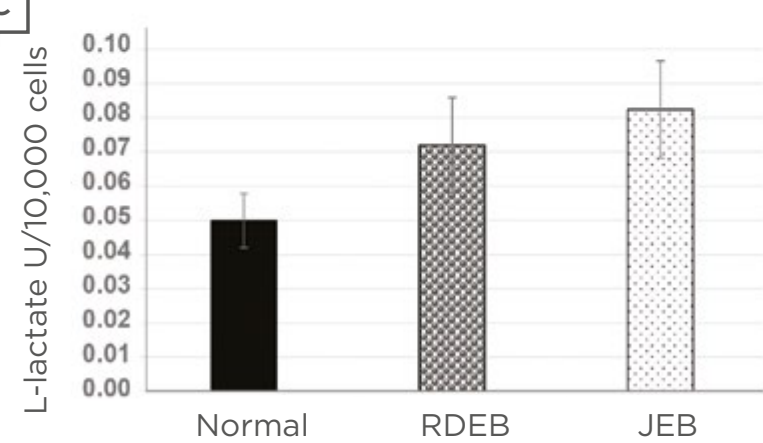

Figure 3: Metabolic profile of recessive dystrophic epidermolysis bullosa and junctional epidermolysis bullosa skin fibroblasts.

A) Representative images of NADH and FAD from RDEB and JEB fibroblasts. Redox ratio images (FAD/ [NADH+FAD]) corresponding to representative NADH and FAD images (bars: $10 \mu M$ ). B) Normalised optical redox ratio (FAD/[NADH+FAD]). Values represent the means over three passages of fibroblasts from each of two controls, six RDEB, and five JEB patients \pm SEM. C) L-lactate production of RDEB and JEB fibroblasts.

Values represent the average over three passages from each of two normal control, six RDEB, and five JEB patient cell lines \pm SEM.

The age ranges for the cell lines: normal (9-13 years), RDEB (1-14 years), and JEB (2 months to 11 years).

Statistical significance was calculated with the Student's t-test: ${ }^{*} p<0.05 ;{ }^{* * *} p<0.001$.

FAD: flavin adenine dinucleotide; JEB: junctional epidermolysis bullosa; RDEB: recessive dystrophic

epidermolysis bullosa.

\section{DISCUSSION}

New data are emerging that mitochondria have critical functions in skin physiology. ${ }^{8}$ RDEB and JEB both involve massive trauma to the skin caused by the loss of structural integrity of the basement membrane. However, the effects on bioenergetic and metabolic status of these conditions have not been studied at the cellular level. Using measurements for OCR, ATP/ADP energy ratio, and mitochondrial morphology, this study has identified a number of important variables in fibroblasts derived from RDEB or JEB patients. Critically, OXPHOS is reduced in RDEB and JEB fibroblasts. The lower basal respiration of EB fibroblasts readily indicates a lower energy output from the electron transport chain. This can reduce the amount of energy available to cells and can be detrimental for cells that carry a large metabolic load, such as fibroblasts involved in the perpetual wound healing found in patients with EB. Paired with this decreased metabolic capability appears to be an increase in the complexity of the mitochondria in EB fibroblasts, which is symptomatic of mitochondrial stress. These overall results are indicative of cell autonomous effects in RDEB and JEB fibroblasts independent of the patient wound status or extracellular environment. Future study is necessary to determine the extent 
to which these cell autonomous effects are impacted by the environmental cues present in EB skin, such as oxidative imbalance and reduced antioxidant enzymes. ${ }^{40}$

\section{CONCLUSION}

EB cells responded to reduced mitochondrial OXPHOS energy production by modestly increasing anaerobic energy production through glycolysis. This was observed through increased production of L-lactate and a reduced redox ratio, while ECAR was only significantly higher in JEB fibroblasts under basal respiratory conditions. This is significant as the cells rely upon glycolysis for energy needs, but still exhibit an energy deficit at the level of ATP availability. The reliance of EB fibroblasts upon glycolysis in a reduced energy state may contribute to the observed range of EB phenotypes. Cells that are better able to adapt or overcome changes in bioenergetic and metabolic states may lead to uneven clinical presentation among patients, or even in different areas of the same patient. One of the treatments for EB patients is bone marrow transplantation. ${ }^{41}$ Transplant outcomes have been shown to be affected by patient and donor mitochondrial haplotype. ${ }^{42}$ Haplotype differences in mitochondrial function and stress response may prove significant as this work has demonstrated the first evidence of altered function and structure of mitochondria in fibroblasts derived from RDEB and JEB patients. EB at its core is more than an extracellular matrix or wounding disorder. This work suggests that systemic response to injury may decide the outcome of cellular and subcellular defects, in turn affecting the severity and treatment response in EB patients. Skin-directed mitochondrial research opens a novel avenue toward the development of EB treatments.

\section{References}

1. Fine JD et al. The classification of inherited epidermolysis bullosa (EB): report of the third international consensus meeting on diagnosis and classification of EB. J Am Acad Dermatol. 2008;58(6):931-50.

2. Fine JD et al. Epidermolysis bullosa and the risk of life-threatening cancers: The National EB Registry experience, 1986-2006. J Am Acad Dermatol. 2009;60(2):203-11.

3. Ashton $\mathrm{GH}$ et al. Recurrent mutations in kindlin-1, a novel keratinocyte focal contact protein, in the autosomal recessive skin fragility and photosensitivity disorder, Kindler syndrome. J Invest Dermatol. 2004;122(1):78-83.

4. Breitenbach JS et al. Transcriptome and ultrastructural changes in dystrophic epidermolysis bullosa resemble skin aging. Aging. 2015;7(6):389-411.

5. Cui $\mathrm{H}$ et al. Oxidative stress, mitochondrial dysfunction, and aging. J Signal Transduct. 2012;2012:646354.

6. Mallipeddi R et al. Increased risk of squamous cell carcinoma in junctional epidermolysis bullosa. J Eur Acad Dermatol Venereol. 2004;18(5):521-6.

7. South AP et al. Understanding the pathogenesis of recessive dystrophic epidermolysis bullosa squamous cell carcinoma. Dermatol Clin. 2010;28(1):171-8.

8. Feichtinger RG et al. Mitochondrial dysfunction: a neglected component of skin diseases. Exp Dermatol. 2014;23(9):607-14

9. Hamanaka RB, Chandel NS. Mitochondrial metabolism as a regulator of keratinocyte differentiation. Cell Logist. 2013;3(2):e25456.

10. Ni-Komatsu L, Orlow SJ. Identification of novel pigmentation modulators by chemical genetic screening. J Invest Dermatol. 2007;127(7):1585-92.

11. Baris OR et al. The mitochondrial electron transport chain is dispensable for proliferation and differentiation of epidermal progenitor cells. Stem Cells. 2011;29(9):1459-68.

12. Huttemann M et al. Regulation of mitochondrial oxidative phosphorylation through cell signaling. Biochim Biophys Acta. 2007;1773(12):1701-20.

13. Houten SM et al. A general introduction to the biochemistry of mitochondrial fatty acid betaoxidation. J Inherit Metab Dis. 2010;33(5):469-77.

14. Lill R et al. Maturation of iron-sulfur proteins in eukaryotes: mechanisms, connected processes, and diseases. Annu Rev Biochem. 2008;77: 669-700.

15. Cheng $Z$ et al. Mitochondria and metabolic homeostasis. Antioxid Redox Signal. 2013;19(3):240-2.

16. Miller WL. Steroid hormone synthesis in mitochondria. Mol Cell Endocrinol. 2013;379(1-2):62-73.
17. Miller WL, Bose HS. Early steps in steroidogenesis: intracellular cholesterol trafficking. J Lipid Res. 2011;52(12):2111-35.

18. Baughman JM et al. Integrative genomics identifies MCU as an essential component of the mitochondrial calcium uniporter. Nature. 2011;476(7360):341-5.

19. De Stefani D et al. A forty-kilodalton protein of the inner membrane is the mitochondrial calcium uniporter. Nature. 2011;476(7360):336-40.

20. Lopez J et al. Mitochondrial apoptosis: killing cancer using the enemy within. $\mathrm{Br} \mathrm{J}$ Cancer. 2015;112(6):957-62

21. Duchen MR. Mitochondria in health and disease: perspectives on a new mitochondrial biology. Mol Aspects Med. 2004;25(4):365-451.

22. Chalut $\mathrm{KJ}$ et al. Light scattering measurements of subcellular structure provide noninvasive early detection of chemotherapyinduced apoptosis. Cancer Res. 2009;69(3):1199-204.

23. Boustany NN et al. Calciuminduced alterations in mitochondrial morphology quantified in situ with optical scatter imaging. Biophys J. 2002;83(3):1691-700.

24. Wilson JD et al. Light scattering from intact cells reports oxidative-stressinduced mitochondrial swelling. Biophys J. 2005;88(4):2929-38. 
25. Passos JF et al. Mitochondrial dysfunction accounts for the stochastic heterogeneity in telomeredependent senescence. PLoS Biol. 2007;5(5):e110.

26. Walsh AJ et al. Optical metabolic imaging identifies glycolytic levels, subtypes, and early-treatment response in breast cancer. Cancer Res. 2013;73(20):6164-74.

27. Varone A et al. Endogenous twophoton fluorescence imaging elucidates metabolic changes related to enhanced glycolysis and glutamine consumption in precancerous epithelial tissues. Cancer Res. 2014;74(11):3067-75

28. Kim HJ, Winge DR. Emerging concepts in the flavinylation of succinate dehydrogenase. Biochim Biophys Acta. 2013;1827(5):627-36.

29. Lehninger AL. Oxidative phosphorylation. Harvey Lect. 1953:49:176-215.

30. Kearney EB. Studies on succinic dehydrogenase. XII. Flavin component of the mammalian enzyme. J Biol Chem. 1960;235:865-77.

31. Sazanov LA. A giant molecular proton pump: structure and mechanism of respiratory complex I. Nat Rev Mol Cell Biol. 2015;16(6):375-88.

32. $\mathrm{Ng} Y Z$ et al. Fibroblast-derived dermal matrix drives development of aggressive cutaneous squamous cell carcinoma in patients with recessive dystrophic epidermolysis bullosa. Cancer Res. 2012;72(14):3522-34.

33. Chance B et al. Oxidation-reduction ratio studies of mitochondria in freeze-trapped samples. NADH and flavoprotein fluorescence signals. J Biol Chem. 1979;254(11):4764-71.

34. Quinn KP et al. Characterization of metabolic changes associated with the functional development of 3D engineered tissues by non-invasive, dynamic measurement of individual cell redox ratios. Biomaterials. 2012;33(21):5341-8.

35. Quinn KP et al. Quantitative metabolic imaging using endogenous fluorescence to detect stem cell differentiation. Sci Rep. 2013;3:3432.

36. Drezek R et al. Autofluorescence microscopy of fresh cervicaltissue sections reveals alterations in tissue biochemistry with dysplasia. Photochem Photobiol. 2001;73(6):636-41.
37. Ramanujam $\mathrm{N}$ et al. Low temperature fluorescence imaging of freezetrapped human cervical tissues. Opt Express. 2001;8(6):335-43.

38. Skala $\mathrm{MC}$ et al. In vivo multiphoton microscopy of NADH and FAD redox states, fluorescence lifetimes, and cellular morphology in precancerous epithelia. Proc Natl Acad Sci. 2007:104(49):19494-9.

39. Skala MC et al. Multiphoton microscopy of endogenous fluorescence differentiates normal, precancerous, and cancerous squamous epithelial tissues. Cancer Res. 2005:65(4):1180-6.

40. Chacón-Solano E et al. Fibroblast activation and abnormal extracellular matrix remodelling as common hallmarks in three cancer-prone genodermatoses. Br J Dermatol. 2019;181(3):512-22

41. Wagner JE et al. Bone marrow transplantation for recessive dystrophic epidermolysis bullosa. N Engl J Med. 2010;363(7):629-39.

42. Ross JA et al. An exploratory analysis of mitochondrial haplotypes and allogeneic hematopoietic cell transplantation outcomes. Biol Blood Marrow Transplant. 2015;21(1):81-8. 